Recepción: 27 de diciembre de 2019

Aprobación: 26 de junio de 2021

\title{
CARTOGRAFÍA DE LA \\ PERCEPCIÓN DE LOS \\ DETERMINANTES SOCIALES \\ DE LA SALUD EN ROSARIO, \\ ARGENTINA
}

CARTOGRAPHY OF THE PERCEPTION OF THE SOCIAL DETERMINANTS OF HEALTH

IN ROSARIO, ARGENTINA

MAPEAMENTO DA PERCEPÇÃO DOS DETERMINANTES SOCIAIS DA SAÚDE EM

ROSÁRIO, ARGENTINA

\section{Daniel Airasca ${ }^{1}$ \\ Guillermina Fritschi ${ }^{2}$}

\section{¿Cómo citar este artículo?:}

Airasca, D. y Fritschi, G. (2021). Cartografía de la percepción de los determinantes sociales de la salud en Rosario, Argentina. Cultura Científica, 19, pp. 140 - 157.

https://doi.org/10.38017/1657463X.734

1 Doctor en Educación, Universidad Abierta Interamericana. Facultad de Medicina y Ciencias de la Salud. Director académico. daniel.Airasca@UAI.edu.ar.

2 Licenciada en Educación, Universidad Abierta Interamericana. Facultad de Medicina y Ciencias de la salud. guillermina.Fritschi@UAI.edu.ar 


\section{RESUMEN}

El estudio tuvo por objetivo analizar y ejercitar la percepción de los Determinantes Sociales de la Salud (DSS) en docentes de las Licenciaturas en Kinesiología y en Producción de Bio-imágenes de la Universidad Abierta Interamericana (UAI), Rosario, Argentina. La metodología desarrollada fue la investigación-acción, las técnicas de recolección de datos elegidas fueron la cartografía social, la observación directa y las entrevistas semiestructurada, la muestra la constituyeron sesenta docentes. El proceso mostró que hay dos tipos de categorías emergentes relativas al proceso de percepción: unas son consecuentes al análisis y la reflexión desde una dimensión que considera a las condiciones socioeconómicas, culturales y ambientales, como la inequitativa distribución de la riqueza, la corrupción y el poder corporativo y otras son efecto del impacto emocional vinculado a la movilidad y la inseguridad que manifiestan percibir los/as docentes en su desplazamiento por la ciudad. Los resultados de esta experiencia indican que los encuentros dialógicos colectivos probaron ser estrategias adecuadas para el ejercicio de la agudeza perceptual, como práctica que promueve la transición de un pensamiento mágico ingenuo a un pensamiento crítico político, y así no solo aumentar el vocabulario e interpretar los problemas más centrales, perturbadores y urgentes que determinan la salud de la población, sino también promover su transformación.

Palabras clave: determinantes sociales de la salud, cartografía social, docentes, percepción.

\section{ABSTRACT}

The study aimed to analyze and exercise the perception of the Social Determinants of Health (SDH) in teachers of Kinesiology and Bio-imaging Production of the Universidad Abierta Interamericana (UAI), Rosario, Argentina. The methodology developed was action research, the data collection techniques chosen were social mapping, direct observation and semi-structured interviews, the sample consisted of sixty teachers. The process showed that there are two types of emerging categories related to the perception process: some are consequent to the analysis and reflection from a dimension that considers socio-economic, cultural and environmental conditions, such as the inequitable distribution of wealth, corruption and corporate power, and others are the effect of the emotional impact linked to mobility and insecurity that teachers perceive as they move around the city. 
The results of this experience indicate that the collective dialogic encounters proved to be adequate strategies for the exercise of perceptual acuity, as a practice that promotes the transition from naive magical thinking to critical political thinking, and thus not only increase the vocabulary and interpret the most central, disturbing and urgent problems that determine the health of the population but also promote their transformation.

Keywords: social determinants of health, social mapping, teachers, perception.

\section{RESUMO}

O objetivo do estudo foi analisar e exercitar a percepção dos Determinantes Sociais da Saúde (DSS) em docentes do Curso de Bacharelado em Cinesiologia e Produção de Bioimagem da Universidade Aberta Interamericana (UAI), Rosário, Argentina. A metodologia desenvolvida foi a pesquisa-ação, as técnicas de coleta de dados escolhidas foram cartografia social, observação direta e entrevistas semiestruturadas, a amostra foi composta por sessenta professo- res. O processo mostrou que existem dois tipos de categorias emergentes relacionadas ao processo de percepção: algumas são consistentes com análises e reflexão a partir de uma dimensão que considera as condições socioeconômicas, culturais e ambientais, como distribuição desigual de riqueza, corrupção e poder corporativo e outras são o efeito do impacto emocional ligado à mobilidade e à insegurança que os professores manifestam quando se deslocam pela cidade. Os resultados dessa experiência indicam que os encontros dialógicos coletivos se mostraram estratégias adequadas para o exercício da acuidade perceptiva, como uma prática que promove a transição do pensamento mágico ingênuo para o pensamento político crítico, e assim não apenas aumentando o vocabulário e interpretando o mais central, Problemas preocupantes e urgentes que determinam a saúde da população, mas também promovem a sua transformação.

Palavras-chave: determinantes sociais da saúde, mapeamento social, professores, percepção. 


\section{INTRODUCCIÓN}

Los Determinantes Sociales de Salud (DSS) son las condiciones en que la gente nace, crece, trabaja y envejece, y son causa de gran parte de las desigualdades sanitarias entre los países y aún dentro de cada país. En otras palabras, se trata de la distribución del poder, los ingresos, los bienes que condicionan el acceso a la atención sanitaria, la escolarización y la educación, así como sus condiciones de trabajo y ocio, tanto como el estado de su vivienda y su entorno físico. La expresión "determinantes sociales" resume entonces el conjunto de factores sociales, políticos, económicos, ambientales y culturales que influyen en el estado de salud (Baum, 2018).

El interés en abordar este tema desde el ámbito de las comunidades universitarias, especialmente las dedicadas a las ciencias de la salud, surge de la vinculación entre los problemas reales que atraviesan a ciudadanos y el trabajo académico. La educación universitaria no puede desvincularse de los problemas sociales a los que se enfrenta la sociedad. Las teorías críticas, inspiradas en Paulo Freire, insisten en que estos problemas no son solo políticos sino inherentemente pedagógicos (Johnson, 1997). Desde esta perspectiva, consideramos que, en el ámbito de la educación superior, las comunidades de aprendizaje deben hacer un esfuerzo por interpretar los cambios del entorno con consecuencias en la salud para, poder intervenir responsablemente sobre las realidades.
Los antecedentes en el estudio del tema coinciden en reconocer que los DSS requieren una amplia gama de acciones que involucran la colaboración de múltiples sectores, entre ellos, la educación, la justicia y el empleo, así como todos los niveles de gobierno (Andermann et al., 2016). En lo que concierne al ámbito de la salud, son los efectores sanitarios quienes están mejor posicionados para promover acciones y operar como catalizadores potenciales del cambio trabajando interdisciplinariamente con las comunidades educativas. Si bien la identificación y toma de conciencia de los DSS son necesarios, no es sin el trabajo complejo de articulación con múltiples saberes y agentes que se puedan reducir las inequidades en la salud y la justicia social que promueve la Organización Mundial de la Salud en sus bases del 2008 (Venkatapuram, 2008).

No obstante, los profesionales de la salud a menudo se sienten impotentes y frustrados cuando se enfrentan a los complejos desafíos sociales y de salud de la gente (Social determinants of health what doctors can do?, 2011). En esos casos evitan involucrarse en los asuntos sociales y prefieren enfocarse en el tratamiento específico y reducir su intervención al asesoramiento sobre el estilo de vida (Braveman, Egerter y Barclay, 2011, p. 7). Además, y este fenómeno no difiere en la población en general, la comprensión de las circunstancias que condicionan la salud en los profesionales sanitarios, regularmente ignora los factores socioestructurales o lo pasan a un segundo plano (Raphael,

\author{
Los Determinantes \\ Sociales de Salud \\ (DSS) son las \\ condiciones en \\ que la gente nace, \\ crece, trabaja y \\ envejece, y son \\ causa de gran \\ parte de las \\ desigualdades \\ sanitarias entre \\ los países y aún \\ dentro de cada \\ país.
}


2016; McIntyre et al., 2013). Considerando solo las conductas individuales (tabaco, dieta, actividad física, etc.) (Shyleyko y Godley, 2013).

Estudios del Instituto Canadiense para la Información de la Salud (2014), estiman que el inequitativo acceso a la atención, sumado a las oportunidades, escasamente aprovechadas, para la promoción de la salud, condicionan las ineficiencias en el sistema de salud. En la misma línea, la Asociación Canadiense de Medicina, reconoce el potencial de las actividades educativas para ayudar a la ciudadanía a enfrentar los desafíos sociales, creando en las comunidades universitarias la necesaria sensibilidad para percibir e identificar esas circunstancias sociales causantes de inequidad (Canadian Medical Association, 2013).

De este modo, entre las principales finalidades de este trabajo está, no sólo determinar la percepción de las DSS, sino desarrollar la agudeza perceptual de los y las docentes, a través de talleres que se llevaron a cabo en las diferentes carreras que conforman la Facultad de Medicina y Ciencias de la Salud de la UAI. Se utilizó la cartografía social para visibilizar a los DSS. Con esta metodología, se puede aprender a enfocar la atención y hacer distinciones respecto a la información recibida del ambiente, con el fin de advertir si lo que se está haciendo, en este caso enseñar, conduce a las metas propuestas.

El decurso cotidiano muestra que la limitación física y cultural de la experiencia sensible, demanda de los docentes un estado de permanente alerta. La percepción no es una simple experiencia sensorial porque posee un significado y una resonancia afectiva (valor); es el rasgo afectivo percibido lo que hace que la percepción sea emocional. La percepción despierta una determinada actitud, una manera de relación práctica con su entorno, ergo promueve la acción (Wittgenstein, 2009). El desarrollo y afinamiento de las habilidades perceptivas hacen que las capacidades de los sujetos varíen.

Entonces, la capacidad para percibir se ejercita mediada por procesos sociales y culturales. Aprender con los demás a explorar el medio y configurar el significado de la experiencia perceptiva, es un ejercicio de reflexión y análisis permanente. Más allá de sus cualidades sensoriales, de la determinación de su diseño y la estabilidad de su forma, la percepción finalmente no solo es modulada por la cultura, sino que se aprende. La incidencia del entorno perfila nuevas formas, descubriendo al mismo tiempo nuevas maneras de percibir y de responder emocionalmente.

\subsection{Determinantes Sociales de la Salud}

Existen distintos modelos para explicar los determinantes de las desigualdades en salud. Aquí, se utilizó el modelo propuesto por la Comisión de Determinantes Sociales de la Salud de la Organización Mundial de la Salud (OMS, 2013), porque tiene en cuenta las circunstancias sociales y ambientales del proce- 
so salud-enfermedad. No se trata de variables sueltas, no relacionadas, sino de procesos que permiten identificar en forma jerarquizada al menos tres determinantes subordinados uno al otro. El primer determinante es el estructural que está dado por la posición social de la persona, así como por los contextos sociales, políticos, económicos y culturales, entre otros; el segundo, es el intermedio y comprende las exposiciones y vulnerabilidades diferenciales de los grupos poblacionales, como el familiar, escolar, laboral o social de la persona y trata de las condiciones materiales en las que viven los individuos, familias y comunidades. Kaplan (2006) insiste en que los recursos materiales influyen en los procesos psicosociales como la falta de control, de apoyo social, la exposición a situaciones de estrés, y también en las conductas que influencian la salud y los procesos biológicos que de todo ello derivan. El tercer determinante es el proximal y está descrito por factores de riesgo personales como la edad, la carga genética, las morbilidades de base, el sexo y la etnia. Es necesario remarcar que categorías como el sexo o el género pueden ser también determinantes estructurales, en la medida que modifiquen la posición social de la persona y se traduzcan en un handicap social o en el acceso y utilización de los servicios de salud. Una forma operativa o metodológica de abordar problemas de salud y/o de calidad de vida, obedece a una postura ontológica y a una intencionalidad epistemológica (Ferrelli, 2015, p. 394), como es el caso que nos ocupa.

\subsection{La cartografía social como forma de acercarnos a las percepciones de los DSS}

La cartografía social es una novedosa forma de intervención que se funda en la investigación-acción, reconociendo al territorio como elemento fundamental de la metodología. Es una ciencia que estudia los procedimientos de obtención de datos sobre el trazado del territorio, para su posterior representación técnica y artística en mapas que conforman la posibilidad de comunicar, en este caso, las percepciones sobre los DSS (Habegger, Mancilla y Serrano, 2006).

Esta técnica requiere del diseño y uso de mapas, fotos, íconos, dibujos y gráficos que facilitan el acercamiento de la comunidad a su espacio geográfico, socioeconómico e histórico-cultural; basándose siempre en la representación social (imagen colectiva) que tienen los miembros de una comunidad de su territorio. Su potencia de transformación social es evidente en tanto pone a disposición de la comunidad los resultados de la investigación que favorece la comprensión de su propia realidad social y sienta las bases para la resolución de los conflictos.

Esta disponibilidad de recursos al inicio del mapeo, que podría pensarse como un marco que limita y acota la acción, constituye en realidad una suerte de trampolín que alienta la construcción colaborativa. Adicionalmente, dinamiza el proceso incorporando una dimensión estética y simbólica al trabajo. El
La cartografía social es una novedosa forma de intervención que se funda en la investigación -acción, reconociendo al territorio como elemento fundamental de la metodología. 
El estudio de la percepción involucra un examen de conciencia de las personas con base en sus emociones $y$ comportamientos con respecto al entorno. uso de estos recursos amplía las metodologías de investigación participativa. Con la incorporación de recursos creativos y visuales, surgen formas ampliadas de comprensión y reflexión, y otorgan sentido a diversos aspectos de la realidad subjetiva, cotidiana, colectiva e histórica.

Numerosas experiencias dan cuenta de su utilidad: en Sacramento y Los Ángeles, una organización multirracial de derechos civiles utilizó el mapeo para el desarrollo del Healthy City (Advancement Project-Healthy City Participatory Asset Mapping, 2012); Fox y colaboradores en Indonesia se valieron del mapeo para el empoderamiento de las comunidades (Fox, Suryanata y Hershock, 2005); Hristrova, Quattrone, Mashhadi y Capra en 2013, estudiaron el impacto de la participación social en el Open Street Map; Giacomo Rambaldi analizó la propiedad intelectual de las leyendas en los mapas en su estudio "Who owns the Map Legend?" (2005) y la Guía de mapeo comunitario del Programa de las Naciones Unidas para el desarrollo urbano social, económica y ambientalmente sustentable en Kenia (United Nations Human Settlements Programme, 2010), entre otros.

\section{METOdOLOGÍA}

El estudio de la percepción involucra un examen de conciencia de las personas con base en sus emociones y comportamientos con respecto al entorno. A los efectos de indagar la percepción de los DSS en los docentes que enseñan en la Universidad Abierta Interameri- cana, se construyó un instrumento basado en el territorio y, para ello, se diseñó un mapa de la ciudad de Rosario (figura 1). Se construyeron iconos de los DSS (figura 2) y las principales emociones representadas con emoticones (figura 3).

Se tomaron como unidad de análisis los claustros docentes de las carreras pertenecientes a la Facultad de Medicina y Ciencias de la salud, de la Universidad Abierta Interamericana de la ciudad de Rosario. La muestra fue seleccionada por conveniencia entre los docentes de carreras de ciencias de la salud (Licenciatura en Kinesiología, Licenciatura en Educación Física y Licenciatura en Producción de Bioimágenes); las técnicas de recolección de datos utilizadas fueron la cartografía social, la observación directa en los talleres y en la realización de entrevistas semiestructuradas.

\subsection{Primera etapa: diseño y producción de recursos}

Consistió en la realización de articulaciones al interior de las carreras de la universidad, con relación a la producción de los insumos necesarios para llevar a cabo los recursos que implica el mapeo y la cartografía social, los iconos y mapas, elementos centrales para poder llevar a cabo los talleres, fueron diseñados por estudiantes del primer año de la carrera de Diseño Gráfico, en el marco de las prácticas de integración en el abordaje de problemas socioprofesionales. Los estudiantes evaluaron y eligieron las propuestas entre los diferentes grupos que conformaron los diseños del mapa, así como de los diferentes íconos 
que servirían para identificar en el territorio a los DSS. Fue relevante el ejercicio de articular la investiga- ción con otros actores institucionales que trabajaban las mismas categorías desde otra perspectiva.

Figura 1. Mapa de la ciudad de Rosario

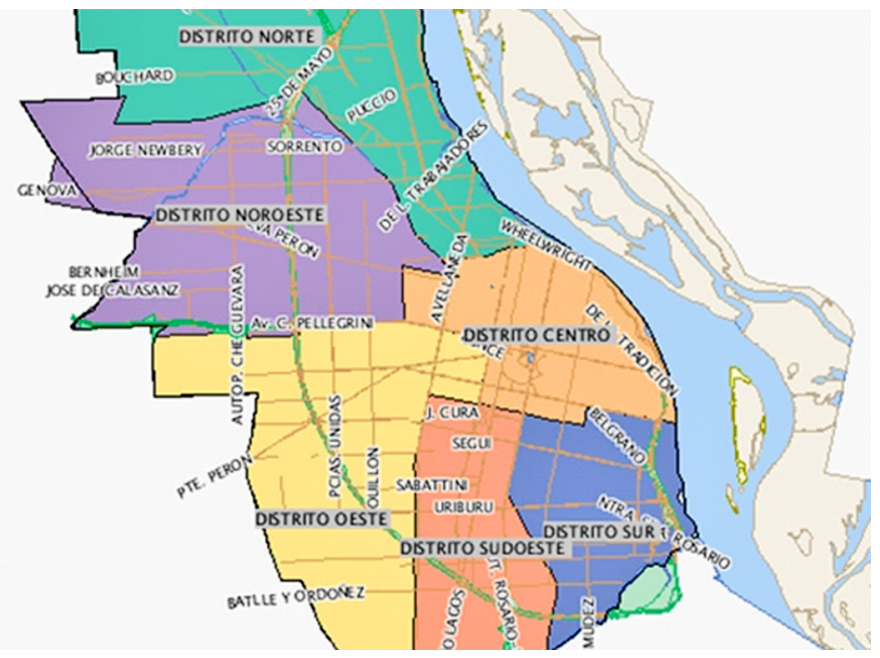

Fuente: elaboración de diseño de Estudiantes de $1^{\circ}$ año.

Figura 2. Pictogramas de DSS

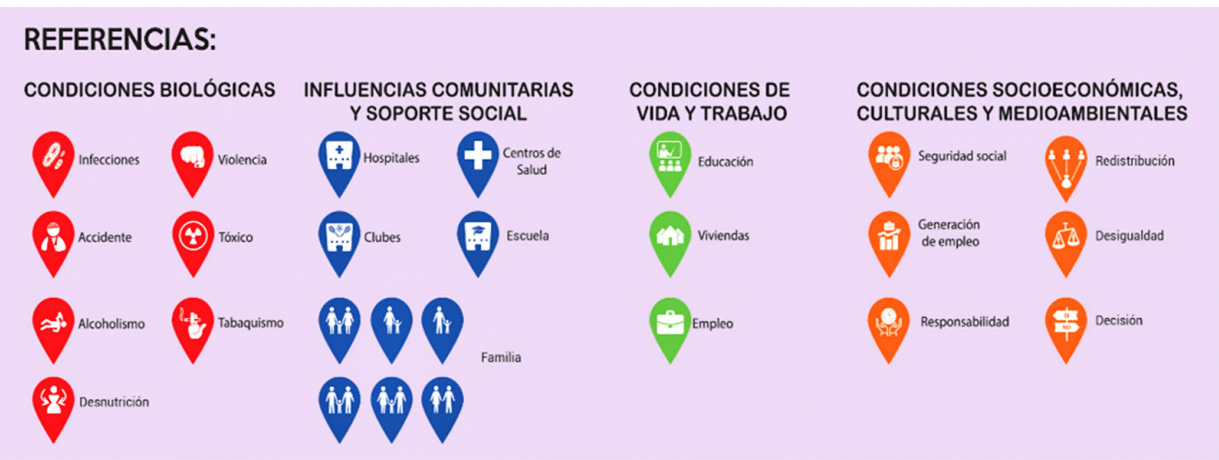

Fuente: elaboración propia de los estudiantes de $1^{\circ}$ año, a partir del modelo de DSS de la OMS.

Figura 3. Pictogramas de las emociones

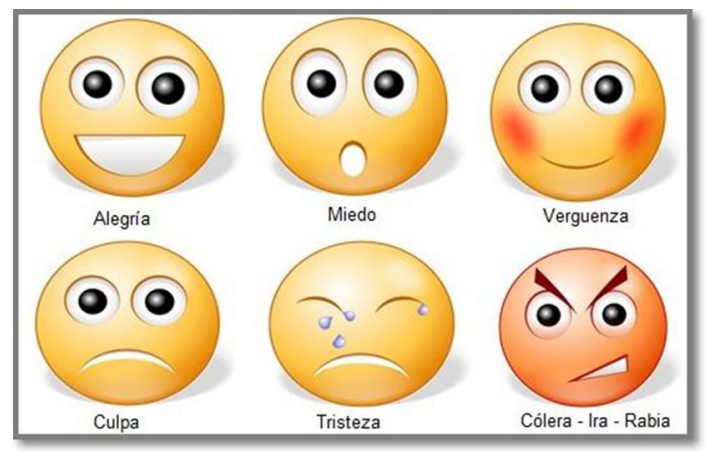

Fuente: Musi, 2019. 
2.2 Segunda etapa: implementación de talleres

El taller fue la técnica que permitió problematizar y reflexionar sobre las percepciones de los DSS para una toma de conciencia en torno a las prácticas cotidianas, los mismos se desarrollaron en las instancias de capacitación pedagógica que propone la universidad; se diseñaron cuatro etapas que permitieron la indagación con base en interrogantes sobre sus vivencias en el territorio y las prácticas de enseñanza, los encuentros/taller comprendieron diferentes tareas: presentación de la propuesta y explicitación del propósito; descripción del territorio habitado representado por la gráfica simplificada sobre una superficie bidimensional plana (figura 1); identificación de los DSS, elección de las emociones (emoticones) que representan una expresión del rostro con el objetivo de aludir a un estado anímico (figura 3) en sus recorridos cotidianos por la ciudad. La pregunta convocante fue: ¿qué sensaciones vivencian en una jornada laboral en el trayecto que circulan desde que salen de su hogar hasta que regresan?

Figura 4. Situando los signos de los DSS en el territorio, mapa Facultad de Medicina y Ciencias de la Salud, UAI, Rosario

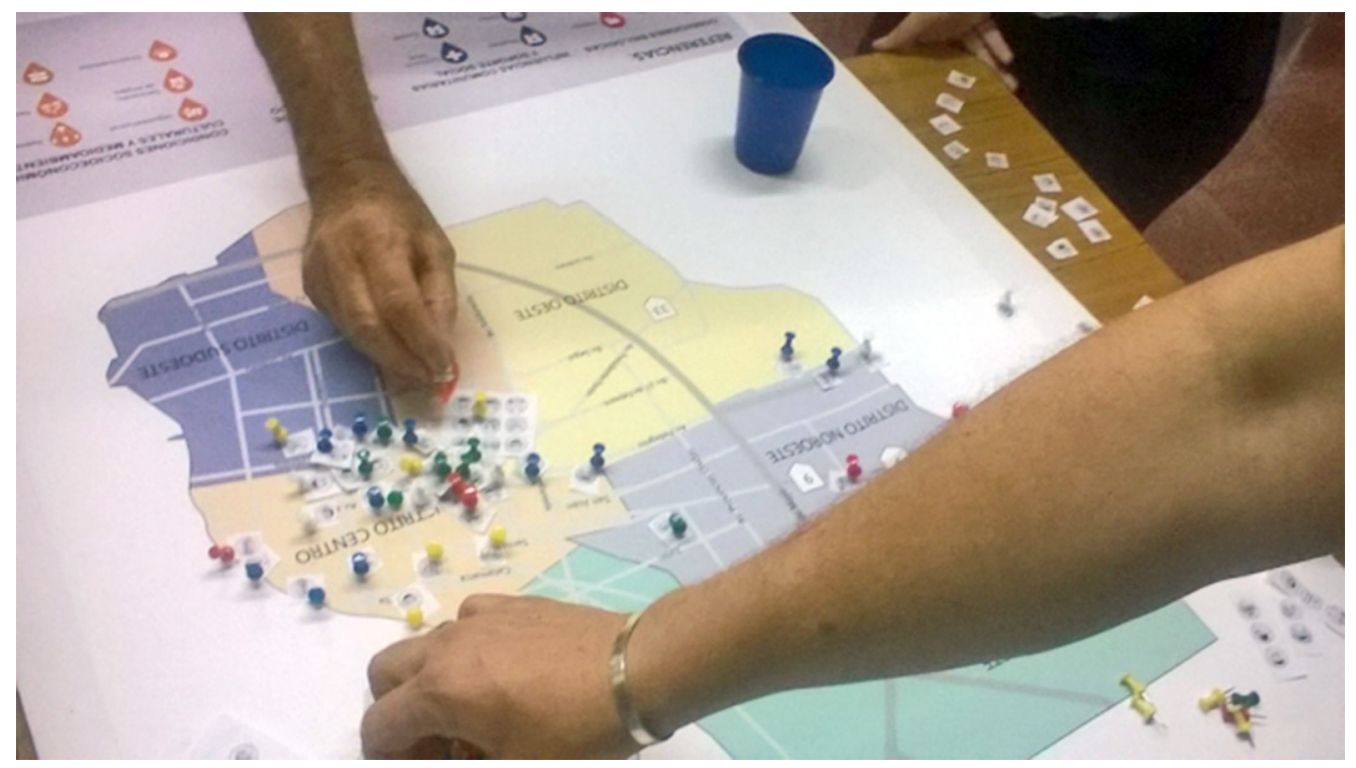

Fuente: elaboración propia.

Reconstrucción de las vivencias territoriales y recorridos individuales y socialización. 
Figura 5. Discutiendo la ocurrencia de los DSS. Mapa Facultad de Medicina y Ciencias de la Salud, UAI, Rosario

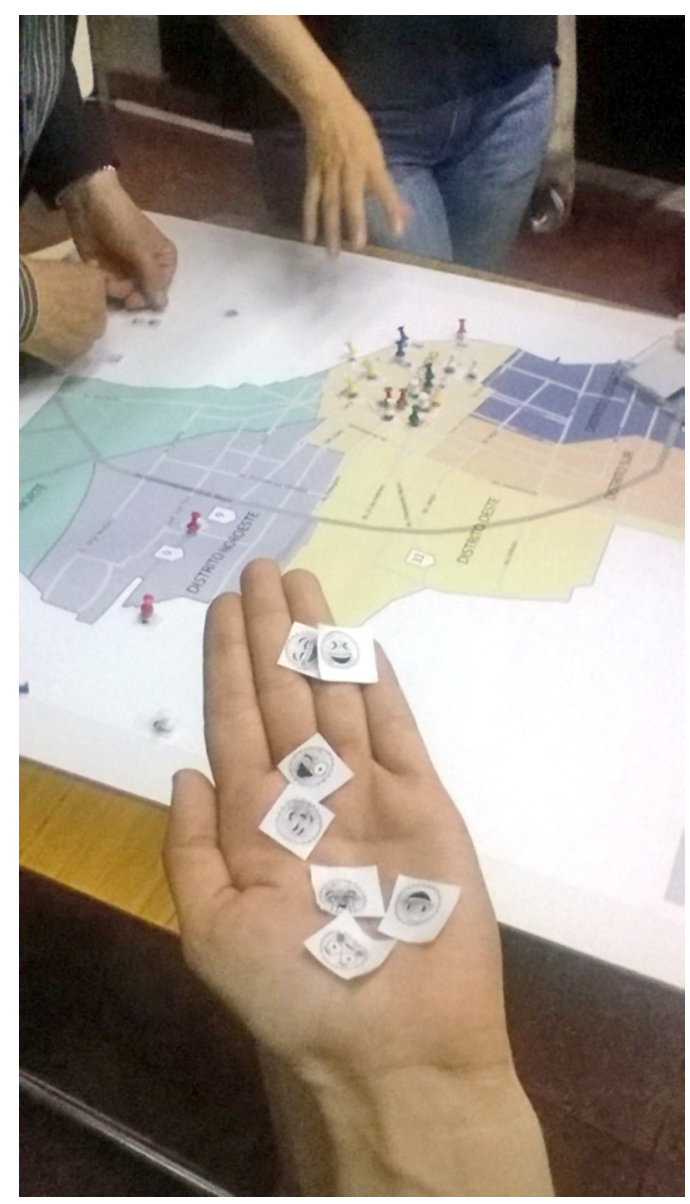

Fuente: elaboración propia.

Como se observa en las fotografías, se señalaron en el mapa los recorridos habituales y lugares de vivencias (figura 4), se reconocieron las percepciones: aromas, sonidos, sensaciones, imágenes, sentimientos y emociones. Se hicieron conscientes las emociones, se escogieron y ubicaron los íconos de la emoción correspondiente, se reconocieron las circunstancias que determinan el estado de salud en el territorio, se eligieron las iconografías correspondientes y se ubicaron sobre el mapa (figura 5).

\subsection{Tercera etapa: grupos de discusión}

Los participantes conformaron pequeños grupos para reflexionar y someter al escrutinio crítico sus propias experiencias en la implementación y el despliegue basados en la cartografía, se facilitó y guió el diálogo con preguntas disparadoras sobre el origen de la percepción personal de los DSS, las vivencias en sus prácticas, los medios de comunicación, el proceso de concienciación; vínculos con las emociones, posibilidad de identificación de 
las emociones y su valoración, así como discusión sobre las estrategias utilizadas en las prácticas de la enseñanza a los fines de abordar las problemáticas contextualizadas; de allí, se trabajó posteriormente en la realización de entrevistas semiestructuradas con quienes accedieron a la conversación.

\section{RESULTADOS Y DISCUSIÓN}

\subsection{Análisis interpretativo de la percepción de los DSS}

El análisis interpretativo es quien habilitó devolver el universo de sentido, que nuestro referente empírico en sí comporta. Así, el trabajo con los docentes permitió permanecer vinculados constantemente a las realidades, el diálogo y el análisis conjunto enriquecieron y profundizaron el trabajo, nadie mejor que la persona involucrada para hablar sobre lo que siente y piensa, aun cuando siempre nos dará su imagen de las cosas, lo que él cree que son, con sus propias subjetividades.

Todo este corpus teórico constituido por los aportes del campo, fueron válidos como relevantes para nuestro trabajo. Se ha "dejado hablar al material recolectado"; así, los sujetos, de entrevista, de observación, permitieron aflorar una teoría propia del "campo", la perspectiva émica (la voz de los sujetos, sus percepciones, su cosmovisión) que al cruzarlo con la teoría, contribuyeron a formular datos, que fueron andamiando el proceso de investigación.
El sesgo crítico de nuestro análisis se puso de manifiesto en las percepciones que contribuyeron a la reproducción de la sociedad y promover la capacidad de reactuar sobre las mismas para revertirlas. Se propuso la crítica como condición irremplazable para cuestionar las percepciones que aporten a la emancipación de los sujetos y a la transformación de la sociedad. Siempre se procuró evitar que los conceptos operaran como definiciones teórico explicativas de los fenómenos, más bien se utilizaron para problematizar el punto de partida teóricamente definido. Allí, resultó fundamental no restringir la indagación bibliográfica a la temática; permanentemente, se estuvo al pendiente de lecturas que podían estar, en mayor o menor medida, cerca de las preocupaciones.

\subsection{El campo tiene su propia teoría}

Esta investigación es de corte cualitativo, se partió de las nociones de los sujetos y de los contextos de producción de sentidos, los mismos han permitido recrear la realidad y particularizarla a su campo de acción e influencia y he aquí la riqueza y ductilidad de una visión no dogmática, que habilitó a seguir conociendo a partir de la interpretación de emergentes inesperados. Estas categorías emergentes, nuevas, no son ajenas a las categorías preconcebidas de análisis: los determinantes estructurales son aquellas condiciones socioeconómicas, culturales y medioambientales "veladas", que constituyen el entorno del sujeto y afecta a su salud y a su 
posibilidad de tener una vida próspera; sin embargo, "emergieron" al forzar los límites de la conciencia y construir sentido tras posteriores procesos de análisis y reflexión.

A continuación, se detallan y discuten los tramos más representativos en relación con los procesos de percepción y discernimiento que se destacaron a partir del análisis de la experiencia realizada en los talleres, grupos de discusión y entrevistas.

\subsection{Inequitativa distribución de la riqueza}

El desigual reparto de la riqueza y la brecha distributiva son circunstancias condicionantes que provocan escasas o nulas reacciones emocionales entre los docentes; su bienestar subjetivo, propio de un estrato social determinado por el nivel de ingresos, le impiden percibir los olores, las imágenes, los sabores y los sonidos de la pobreza, solo sienten desagrado y molestia cuando "El cartonero que empuja su carro cirugeando entre la basura, es una molestia a la circulación vehicular de la gente que trabaja" (dato obtenido en la descripción que realizaban al momento de marcar el recorrido de salida y llegada al trabajo).

Sus opiniones fluctúan en coherencia con su perfil ideológico, “(...) había un piquete de la CCC cortando el tránsito, ite paran la ciudad!" (dato del campo de otro docente). El taller de cartografía fue una usina del pensamiento. Así, sensaciones que inicialmente se expresaban inconscientemente con emoticones de enojo y rechazo ante la falta de iniciativa y espíritu competitivo de la gente vulnerable: "son todos choriplaneros" (dato del campo); fueron, tras lecturas compartidas y diálogo reflexivo, mudando y haciendo consciente las sensaciones primarias en un espiral constructivo que las dotó de sentido, otorgando valor a la determinación estructural.

\subsection{Corrupción}

La aprehensión de que la corrupción es un fenómeno nocivo, vasto, diverso y global, no es generalizada en todos los grupos, la mayoría de los docentes piensan la corrupción como actos perversos, ilegales o moralmente malos, casi siempre cometidos por autoridades o servidores públicos, pero no en acciones diarias de convivencia. "Estacionarse en doble fila, arrojar desperdicios en la vía pública, doblar a la izquierda, tirarse la clase", no aparecen como actos de corrupción. Solo "son actos de compensación o revancha", por lo que "roban" o por lo "mal que me pagan" (dato del campo).

Parecería que no hay conciencia de cómo las acciones a nivel micro-social tienen consecuencia a mayor escala. "Llegar 15 minutos tarde no es ser corrupta” (dato del campo), y puede tener razón, dada la idea generalizada del término. Tras una larga y acalorada discusión, comenzó a vislumbrarse que algunos actos aparentemente "triviales" violentan las formas de vivir en lo público. "El sistema es corrupto" (dato del campo), soslayando que las personas integran el sistema y verificando que las faltas privadas se visibilizan menos que las públicas. 
El aporte teórico ayuda a descubrir una de las características de la percepción ¿Cuál es la selectividad que hace que los estímulos rutinizados pasen a un segundo plano como conciencia práctica? El proceso de análisis crítico descubre que si existe corrupción pública es consecuencia de la inmoralidad privada, no sorprende la tolerancia hacia la corrupción ciudadana, al punto de considerarla como algo "normal", es un rasgo que podríamos vincular con la idiosincrasia argentina, Georges Clemenceau, primer ministro en la Francia de la Tercera República visitó Argentina en 1910 y dijo: "Argentina crece gracias a que sus políticos y gobernantes dejan de robar cuando duermen". Ya se decía que el país es destruido de día y se reponía de noche.

\subsection{Poder corporativo}

La percepción valorativa que manifiestan los docentes sobre el vínculo entre el poder corporativo transnacional y el impacto socioambiental, es esquiva y muy superficial, no se divisa con claridad el entramado político, económico, jurídico y cultural logrado por las compañías multinacionales, al amparo de las políticas neoliberales para dominar la sociedad; la acumulación del poder y ganancias de las grandes corporaciones fue uno de los DSS más abstrusos y difíciles de detectar.

La oportunidad apareció cuando se mencionaron los hábitos saludables individuales, surgió una suerte de preocupación exagerada por la salud puesta de manifiesto en hábitos y conductas propias de un estilo de vida saludable. "Descanso suficiente, actividad física regular, hidratación adecuada, alimentación sana, suplementación nutricional”, etc. son solo algunos de los hábitos y conductas relatados para mantener el cuerpo estable y aprovechar sus potencialidades.

Se sometió a la crítica esta estrategia de promoción de salud hegemónica basada en el autocontrol, la autonomía y la responsabilidad individual. Sin despreciar su real valor, se trató de encontrar los efectos de su utilización indiscriminada y sus vínculos con aspectos socioculturales indeseables relativos al individualismo, el consumismo y el control social, siempre funcionales a intereses corporativos.

\subsection{Inseguridad y violencia}

El aspecto más referido por los docentes es el robo de domicilios y de automóviles particulares, y los arrebatos y robos callejeros, así como la violencia implementada en estos, algunos relatan que viven con miedo y no solo a ser robados o asaltados, sino también ante situaciones que no se catalogan como delitos; se trata de comportamientos antisociales que configuran la inseguridad en el ámbito urbano. La percepción es la dimensión subjetiva de la inseguridad, es la estimación que cada uno hace sobre el grado de riesgo al que está expuesto, y a veces con independencia de los hechos concretos de violencia y los delitos.

La percepción de inseguridad es una construcción social que condiciona la calidad de vida de los ciudadanos, generando, por ejem- 
plo, conductas de autoprotección, como adoptar medidas de seguridad en el hogar o cambios de hábitos en relación con las actividades cotidianas, "Cuando llego a casa, llamo por teléfono para que me abran la reja y el portón" (dato de campo).

Las impresiones primarias de la mayoría de los docentes, achacan unánimemente la responsabilidad a la falta de autoridad representada por la policía, la gendarmería y el sistema judicial, "nunca están y cuando están, están reunidos usando el celular" (dato del campo). Algunos sostuvieron el debate criticando este modelo de seguridad ciudadana, no solo basados en teorías anarquistas, que pusieron en dudas el monopolio de la coerción por parte del Estado, sino también por la desconfianza en el accionar policial "No hicimos la denuncia porque nunca pasa nada" (dato del campo).

El rescate de teoría y la reflexión conjunta puso en dudas la irresponsabilidad individual y el paternalismo del Estado otorgando al ciudadano el papel más importante en la seguridad pública, y no solo, legitimando democráticamente las anteriores formas de autoridad, sino, y antes bien, moderando sus propias acciones y denunciando actividades ilícitas; este último rasgo, enraíza nuevamente con la cultura argentina, en 1833, Darwin luego de una corta estancia en Buenos Aires describió: "Los habitantes respetables del país ayudan invariablemente al delincuente a escapar, parecería que piensan que el hombre ha pecado contra el gobierno y no contra ellos mismos". La impunidad ya era bien vista y la falta de sanción una costumbre.

\subsection{Transporte y movilidad}

Los docentes decididamente marcan con emoticones de enojo la irritabilidad recordada ante la pérdida de tiempo y estrés causado por conducir inmersos en una masa vehicular excesiva a la hora de desplazarse, "... parados en doble fila para que desciendan los hijos que van a la escuela", "los taxis levantan pasajeros en mitad de la calle", "Qué peligrosos los motociclistas que van por la bicisenda" (datos del campo), las consecuencias de la calidad del transporte relatadas y vinculadas a la irritación tienen que ver con la frustración que genera la congestión e impide el desplazamiento deseado.

No hay respuesta emocional perceptiva por la contaminación acústica y del aire, ni tampoco se hace referencia a los índices de siniestralidad y mucho menos por la ocupación y fragmentación del territorio, que limitan o imposibilitan la utilización de las calles como algo más que canales de transporte; estos problemas que, por su magnitud y gravedad, afectan la calidad de vida de todos los habitantes de la ciudad, generan una incoherente y escasa crítica, muestra de su aceptación en una sociedad que, paradójicamente, ante manifestaciones sociales con repercusiones colectivas de mucho menor impacto -narcotráfico- presenta una clara intolerancia. 
Una primera aproximación a los datos recolectados, permite señalar que hay dos modalidades de percepciones: una con carga emocional; y, la otra, carente de afecto. Si bien la diferencia es gradual, lo que las distingue es el nivel de significación o relevancia para el perceptor, el nivel de significancia depende de los rasgos singulares del perceptor y de anteriores experiencias exploratorias. En este sentido, los procesos de reflexión promovieron un acercamiento más consciente a la realidad a quienes participaron de los talleres.

Así entonces, las categorías emergentes relativas al proceso de percepción no emocional de los DSS, fueron la inequitativa distribución de la riqueza, la corrupción y el poder corporativo. Las otras son efecto del impacto emocional: inseguridad y transporte, pero también la desidia y la anomia social, los análisis realizados y los datos relevados a partir de la cartografía social de la ciudad de Rosario, desde la perspectiva de los docentes, permitió visibilizar espacialmente las circunstancias de los condicionantes de la salud más acuciantes, centrales y perturbadoras del lugar, identificando a los responsables, reflexionando sobre conexiones con otras temáticas remotas y señalando las consecuencias de éstas.

\section{CONCLUSIONES}

La complejidad de la realidad sanitaria actual obliga a construir nuevas estrategias e instrumentos de análisis con los que se puedan in- terpretar las tendencias que rigen la configuración de la salud de la población; de esta forma, la cartografía social se descubre como una adecuada herramienta de planificación y transformación social, basada en la investigación acción-participativa, que facilita el manejo de la información espacial mediante el impacto visual. Esta manera de presentación gráfica de la información, ilustró y presentó las relaciones entre los datos y actuó recursivamente, en tanto facilitó a los participantes de la investigación a mejorar la comprensión de relaciones resultantes de los cambios del mundo actual y analizar sus consecuencias.

Este proceso de investigación acción-participativa, entendido y practicado como un proceso dinámico de indagación y reflexión, amplió el horizonte perceptivo de los docentes al momento de opinar sobre la relación entre responsabilidad individual y responsabilidad social en salud. Se pudo establecer un nexo entre las conductas y estilos de vida singulares (determinantes intermedios) con los contextos sociales (determinantes estructurales) que los modelan y limitan. De esta forma, problemas de salud como el tabaquismo, la desnutrición, el alcoholismo, la exposición a agentes infecciosos y tóxicos, la violencia y los siniestros viales, se subordinan a macrodeterminantes como el nivel de acceso a servicios básicos, educación, empleo, vivienda que traducen la distribución del ingreso, la creencia extendida, funcional a 
la salud hegemónica, que carga las tintas en el accionar individual, se fue decantando en favor de una visión ecológica que la supedita a la responsabilidad social.

Los DSS explican la mayor parte de las inequidades sanitarias, es el contexto social, económico y político el que determina el estado de salud y de desarrollo de una población. Las desigualdades en salud y el bienestar de la ciudadanía, son condicionados por el poder político y el tipo de políticas macroeconómicas y sociales (Comisión para Reducir las Desigualdades Sociales en Salud en España 2009, 2015).

Los DSS como el poder corporativo y la inequitativa distribución de la riqueza, se invisibilizan dentro de la estructura, son de difícil percepción en la vida cotidiana, solamente cuando devienen categorías de análisis desde una perspectiva ecológica, holista y compleja, muestran su vinculación cuanto no íntima, sino determinante con el bienestar de la población.

Para los docentes, es de gran utilidad el ejercicio de percepción de las circunstancias sociales que condicionan la salud de la ciudadanía en claves de problemas que pueden llegar a convertirse en una oportunidad pedagógica y generar junto a los estudiantes soluciones creativas para su transformación, la propuesta de cambio inicia con el abordaje académico de tales circunstancias socioambientales y su tratamiento es ineludiblemente político.
Tal como se identificó anteriormente, la participación en los talleres de cartografía significó una novedosa experiencia que derivó en otras formas de sentir y pensar, la discriminación de la atención, el rescate de la memoria y el razonamiento dialógico, son los ingredientes que modulan los inputs sensoriales y derivan en nuevos conocimientos. La opción de reformular y volver a etiquetar la emoción, implicó cambiar la interpretación de lo que está pasando, constituyendo el primer paso para afrontar las situaciones de una forma distinta.

Es la oportunidad, también, para tomar conciencia de la misión docente, asumir con optimismo estrategias que hagan práctica la esperanza y conviertan la equidad en el fundamento de la pedagogía, o al menos, tratar de ayudar a comprender al capitalismo, pero no como dominación, sino desde la perspectiva de su crisis, de sus contradicciones, debilidades, y, por sobre todo las cosas, entender cómo nosotros mismos somos esas contradicciones.

Los encuentros dialógicos colectivos probaron ser estrategias adecuadas para el ejercicio de la agudeza perceptual, como práctica que promueve la transición de un pensamiento mágico ingenuo a un pensamiento crítico político, y así no solo aumentar el vocabulario e interpretar los problemas más centrales, perturbadores y urgentes que determinan la salud de la población, sino también promover su transformación. 


\section{REFERENCIAS}

Advancement Project-Healthy City. (2012). Participatory Asset Mapping. www. advancementprojectca.org/

Andermann, A., y CLEAR Collaboration (2016). Taking action on the social determinants of health in clinical practice: a framework for health professionals. CMAJ: Canadian Medical Association journal, 188(17-18), E474-E483. https://doi.org/10.1503/cmaj. 160177

Braveman, P., Egerter, S., y Barclay, C. (2011). Income, Wealth and Health. Princeton, Robert Wood Johnson Foundation. https://www.rwjf.org/en/library/ research/2011/04/how-social-factors-shape-health 1 .html

Baum, F. (2018). People's health and the social determinants of health. Health Promot J Austral, 29, 8-9. doi:10.1002/hpja.49

Canadian Medical Association (2013). Health equity and the social determinants of health: a role for the medical profession. CMA. www.cma.ca/Assets/assets-library/document/en/advocacy/PD13-03-e.pdf.

Commission on Social Determinants of Health. Final report. (2008). Closing the gap in a generation: health equity through action on the social determinants of health. CSDH. http://whqlibdoc.who.int/publications/2008/9789241563703_eng.pdf

Comisión para Reducir las Desigualdades Sociales en Salud en España. (2015). Avanzando hacia la equidad. Propuesta de Políticas e Intervenciones para reducir las desigualdades Sociales en salud en España. https://www.mscbs. gob.es/profesionales/saludPublica/prevPromocion/promocion/desigualdadSalud/docs/Propuesta_Politicas_Reducir_Desigualdades.pdf

Fox, J., Suryanata, K., y Hershock, P. (eds.). (2005). Mapping communities. Ethics, values, practice. East-West Center. https://www.eastwestcenter.org/publications/mapping-communities-ethics-values-practice

Ferrelli, R.M. (ed.) (2015). Equidad en salud desde un enfoque de determinantes sociales. http://sia.eurosocial-ii.eu/files/docs/1457517999-DT_39-EQUIDAD\%20OK2b.pdf

Giddens, A. (1995). La constitución de la sociedad. Bases para la teoría de la estructuración. Amorrortu editores.

Habegger, S., Mancilla, J., y Serrano, E. (2006). El poder de la cartografía del territorio en las prácticas contra-hegemónicas. Instituto Paulo Freire. https:// es.scribd.com/document/162754996/Habegger-El-poder-de-la-Cartografia-Social-en-las-practicas-contrahegemonicas-o-La-Cartografia-Social-como-estrategia-para-diagnosticar-nuestro-territ

Hristrova, D., Quattrone, G., Mashhadi, A., y Capra, L. (2013). The life of the party: Impact of social mapping in OpenStreetMap. Association for the Advancement of Artificial Intelligence. www.aaai.org/ocs/ index.php/icwsm/ icwsm 13/paper/ view/6098. 
Instituto canadiense para la información de la salud. (2014). Measuring the level and determinants of health system efficiency in Canada. www.secure.cihi. $\mathrm{ca} /$ freeproducts/HSETechnicalReportENweb.pdf

Johnson, R. (1997). Reinventing Cultural Studies: Remembering for the Best Version. En: Elizabeth Long (ed.), From Sociology to Cultural Studies. Malden, Massachusetts: Basil Blackwell. https://www.wiley.com/en-ar/From+Sociology+to+Cultural+Studies: + New + Perspectives-p-9781577180128

Kaplan, G. (2006). Social Determinants of Health. International Journal of Epidemiology, 35(4), 1111-1112. DOI: 10.1093/ije/dyl121

McIntyre, L., Shyleyko, R., Nicholson, C., Beanlands, H., y McLaren, L. (2013). Perceptions of the social determinants of health by two groups more and less affiliated with public health in Canada. National Library of Medicine. https://www.ncbi.nlm.nih.gov/pmc/articles/PMC3851988/

Musi, A. (2019). Formación en neuro- psico- educación. Asociación educar para el desarrollo humano. www.asociacioneducar.com/monografias/monografia-neurosicoeducacion-alicia.mussin.pdf.

OMS. (2013) The economics of social determinants of health and health inequalities: a resource book. https://apps.who.int/iris/bitstream/ handle/10665/84213/9789241548625_eng.pdf; jsessionid = C CD58A7D485954659BBEAB0F8A20CB5B? sequence $=$.

Rambaldi, G. (2005). Who Owns the Map Legend? URISA, Journal of the Urban and Regional Information Systems Association, 17(1), 5-13. https://research.wur.nl/en/publications/who-owns-the-map-legend

Raphael, D. (2016). Social Determinants of Health, Canadian Perspectives (3. ${ }^{\text {rd }}$ Ed.). https://www.researchgate.net/publication/301695606_Social_Determinants_of_Health_Canadian_Perspectives_3rd_Edition

Rosario mapas: Mapa de Rosario. Recovered from: http://www.rosariomapas. $\mathrm{com} / .777$

Shyleyko, R., y Godley, J. (2013). Post-secondary students' views on health: Support for individual and social health determinants. Alberta Journal of Educational Research, 58(4), 547-564. https://journalhosting.ucalgary.ca/ index.php/ajer/article/view/55578

Social determinants of health - what doctors can do? (2011). https://www. paho.org/hq/dmdocuments/2012/Social-Determinants-Doctors-Eng.pdf

United Nations Human Settlements Programme. (2010). Community mapping guide. A youth community mapping toolkit for East Africa (Vol. 3). https:// issuu.com/unhabitat/docs/community_mapping_guide/22

Venkatapuram, S. (2008). World health report 2008. Primary health care: now more than ever. OMS. www.who.int/whr/2008/whr08_en.pdf

Wittgenstein, L. (2009) Sobre la certeza. Gedisa 